\title{
The biology of hypoxia: the role of oxygen sensing in development, normal function, and disease
}

\author{
Amato J. Giaccia, ${ }^{1,4}$ M. Celeste Simon, ${ }^{2}$ and Randall Johnson ${ }^{3}$ \\ ${ }^{1}$ Department of Radiation Oncology, Stanford University School of Medicine, Stanford, California 94305, USA; \\ ${ }^{2}$ Abramson Family Cancer Research Institute and Howard Hughes Medical Institute, University of Pennsylvania, \\ Philadelphia, Pennsylvania 19104, USA; ${ }^{3}$ Division of Biological Sciences, University of California at San Diego, \\ San Diego, California 92093, USA
}

The ability to sense and respond to changes in oxygen is essential for the survival of prokaryotic and eukaryotic organisms. Oxygen-sensing mechanisms have been developed to maintain cell and tissue homeostasis, as well as to adapt to the chronic low-oxygen conditions found in diseases such as cancer. This report on the first Keystone Meeting on the Biology of Hypoxia will summarize our current understanding of key genes and pathways involved in oxygen sensing that are required for normal development and that are dysregulated in disease states. It will also comment on future directions for this exciting field.

Molecular oxygen is essential for the development and growth of multicellular organisms. Mammals have evolved a sophisticated physiological network to maintain oxygen homeostasis at the tissue level that involves the capture, binding, transport, and delivery of molecular oxygen. One of the critical aspects of this network is the ability to sense and respond to low-oxygen conditions. This conference brought together, for the first time, researchers from a broad range of disciplines to focus on the molecular and physiological mechanisms of oxygen sensing and their roles in normal development, pathological conditions, and cancer. Because our understanding of the mechanisms of oxygen sensing and the effects of oxygen in different model systems has rapidly increased in the last decade, this conference offered an important opportunity to present different perspectives on critical issues that will drive the field forward in the future. Some of the emerging themes in oxygen sensing are discussed below.

\section{Molecular pathways involved in oxygen sensing}

For cells to adapt to hypoxic conditions, they must be able to sense changes in oxygen and respond accordingly

[Keywords: Hypoxia; HIF; hydroxylases; ion channels]

${ }^{4}$ Corresponding author.

E-MAIL giaccia@stanford.edu; FAX (650) 723-7382.

Article and publication are at http://www.genesdev.org/cgi/doi/10.1101/ gad.1243304.
(Fig. 1). The initiation of these responses can be rapid and involve transcriptional and posttranscriptional mechanisms. Although we now have gained great insight into how cellular signal transduction pathways are regulated by hypoxia, the mechanisms of how cells sense changes in oxygen have only recently been elucidated. The current candidates for oxygen sensors include the prolyl hydroxylase family of enzymes that require molecular oxygen for activity, the NAD $(\mathrm{P}) \mathrm{H}$ oxidase family of enzymes that reduce reactive oxygen species (ROS), oxygen sensitive ion channels, and the electron transport chain.

\section{Reactive oxygen species and the mitochondria}

Paul Schumacker (University of Chicago) proposed that hypoxia paradoxically stimulates ROS release from the mitochondria that can subsequently regulate the transcriptional and posttranslational response to low-oxygen conditions (Schumacker 2003). Electron transport in the mitochondria is mediated by protein-bound redox centers such as succinate-coenzyme Q reductase (complex II) and Coenzyme Q-cytochrome c reductase (complex III). He proposed that ROS production in the mitochondria occurs primarily within complex III, although other sites such as complex II may also generate ROS. In the generation of ubiquinol from ubiquinone in the mitochondria, a free radical (ubisemiquinone) is normally generated during the electron-transport process. This radical can potentially donate its unpaired electron to $\mathrm{O}_{2}$, thereby generating superoxide. During hypoxia, the process of ROS generation from that site can be amplified. In contrast, when mitochondrial electron transport is inhibited at a more proximal site by inhibitors such as rotenone, DPI, or myxothiazol, the ubiquinone pool becomes fully oxidized and ROS generation at complex III is abrogated. According to this model, the ROS production at complex III increases during hypoxia, and these oxidants trigger signaling pathways. Evidence in support of this model includes the observations that oxidant stress increases under hypoxia as determined by oxidation of fluorescent probes, decreases reduced glutathi- 
Physiologic Response Pathways to Hypoxia

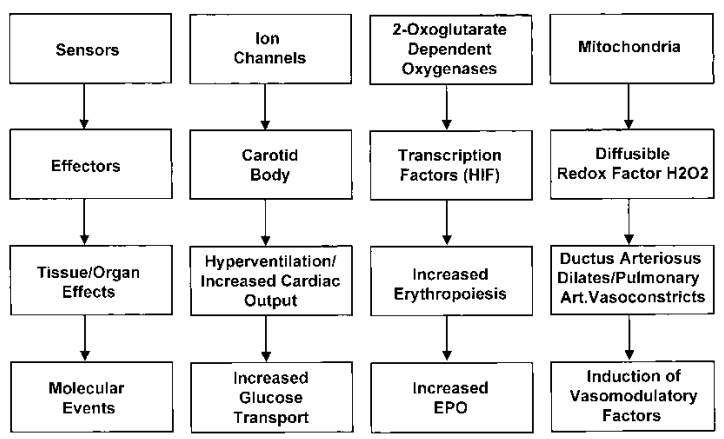

Figure 1. Physiologic response pathways to hypoxia. For details, see text.

one pools, increases radicals detected by ESR spectroscopy, and decreases reduced cysteine (Waypa and Schumacker 2002). Inhibition of ROS under hypoxia inhibits some transcriptional responses to hypoxia. In addition, treatment of cells with oxidants under normoxic conditions induces a hypoxic response. Studies by Navdeep Chandel (Northwestern University) suggested that ROS play a more important role at hypoxic oxygen tensions than at severe anoxic oxygen tensions in protein stabilization and gene regulation (Schroedl et al. 2002). Celeste Simon (University of Pennsylvania) raised the possibility that there may be different oxygen sensors that depend on the level of oxygen (e.g., hypoxia vs. anoxia), a rather intriguing concept. The details of how the generation of ROS directly or indirectly affects gene and protein expression are certainly a challenge for future studies.

\section{Protein synthesis and the unfolded protein response}

Previous studies have suggested that hypoxia inhibits macromolecule synthesis in an oxygen-dependent manner to conserve energy utilization (Heacock and Sutherland 1990). Celeste Simon described the effect of hypoxia on the mammalian target of rapamycin (mTOR) pathway (Arsham et al. 2003). Hypoxia rapidly and reversibly triggers hypophosphorylation of $\mathrm{mTOR}$ and its effectors 4E$\mathrm{BP} 1, \mathrm{p} 70 \mathrm{S6K}, \mathrm{rpS6}$, and eukaryotic initiation factor 4G. Hypoxic regulation of these translational control proteins was shown to be independent of Akt/protein kinase $\mathrm{B}$ and AMP-activated protein kinase phosphorylation, ATP levels, ATP:ADP ratios, and hypoxia-inducible factor-1 (HIF-1). Her studies suggested that hypoxia represses mTOR as if the cell was exposed to rapamycin. These data suggest that mTOR may act as an oxygen sensor and directly link decreased oxygen levels with inhibition of protein translation. She raised the point that further understanding of how translation is regulated under hypoxic conditions is necessary.

In addition to TOR inhibition, Costas Koumenis and Brad Wouters presented data to indicate that severely hypoxic cells can also control translation through the activation of the endoplasmic reticulum kinase PERK, and phosphorylation of translation initiation factor
eIF2 $\alpha$ (Koumenis et al. 2002). The activation of PERK suggests that another way a cell senses changes in oxygen is through endoplasmic reticulum stress. The induction of the UPR pathway is essential for cell survival under hypoxic conditions, as disruption of any of the endoplasmic reticulum transmembrane-signaled pathways sensitizes cells to hypoxia-induced death, and abolishes tumor growth.

Direct sensing of changes in oxygen

by the prolyl hydroxylase family of enzymes

HIF-1 is composed of two subunits, an oxygen-sensitive HIF- $1 \alpha$ subunit and a constitutively expressed HIF-1 $\beta$ subunit (Wang and Semenza 1995; Fig. 2). Both HIF-1 $\alpha$ and HIF-1 $\beta$ are members of the basic helix-loop-helix Per/Sim/Arnt (HLH-PAS) family of transcription factors. In contrast to the constitutively expressed HIF-1 $\beta$ subunit, HIF- $1 \alpha$ is an oxygen labile protein that becomes stabilized in response to hypoxia, iron chelators, and divalent cations. Peter Ratcliffe (Oxford University) discussed the regulation of HIF by oxygen-dependent hydroxylation at specific prolyl and asparaginyl residues within the HIF- $1 \alpha$ and HIF- $2 \alpha$ subunits (Schofield and Ratcliffe 2004). Using elegant genetic approaches in Caenorhabditis elegans, he and his coworkers showed that the enzymes that hydroxylate HIF- $1 \alpha$ and HIF- $2 \alpha$ belong to a family of 2-oxoglutarate-dependent dioxygenases that possess a $\beta$-barrel "jelly-roll" conformation, which aligns a 2-histidine-1-carboxylate iron coordination motif at the catalytic center. At least three prolyl hydroxylases have been found to hydroxylate HIF on unique proline residues, and have been termed PHD-1, PHD-2, and PHD-3. Under normoxic conditions, hydroxylation of

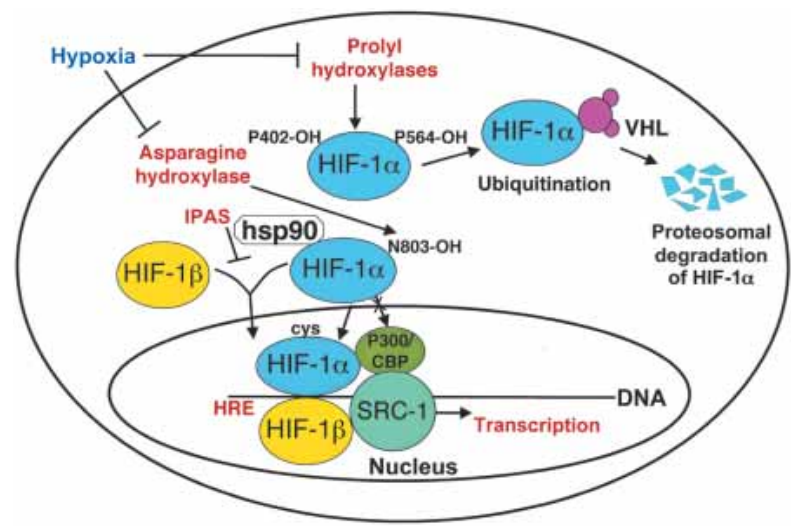

Figure 2. Mechanisms of HIF- $1 \alpha$ regulation under aerobic and hypoxic conditions. Under aerobic conditions, HIF- $1 \alpha$ is hydroxylated on proline 402 and proline 564. The proline hydroxylations are necessary for binding to VHL and ubiquitin-mediated degradation by the proteasome. The asparagine hydroxylation prevents binding to $\mathrm{p} 300 / \mathrm{CBP}$. A splice derivative of HIF- $3 \alpha$ called IPAS, as it only possesses the PAS domain, competes for HIF-1 $\beta$ binding. The TAD of HIF- $1 \alpha$ binds p300/CBP and other coactivators such as SRC-1. HIF- $1 \alpha$ and HIF- $1 \beta$ both translocate to the nucleus to transactivate genes such as VEGF that possess hypoxia responsive elements (HREs). 
HIF- $1 \alpha$ on these different proline residues is essential for HIF proteolytic degradation by promoting interaction with the von Hippel-Lindau tumor-suppressor protein (pVHL) through hydrogen bonding to the hydroxyproline-binding pocket in the pVHL $\beta$-domain. As oxygen levels decrease, hydroxylation of HIF decreases; HIF- $1 \alpha$ then no longer binds pVHL, and becomes stabilized. Whereas hydroxylation of each proline can act independently to promote degradation of HIF, biochemically, the proline 564 site of HIF- $1 \alpha$ has a greater affinity for PHDs than the 402 site. Denise Chan (Stanford University) presented genetic evidence that hydroxylation of one site can influence hydroxylation of the other site, suggesting a more complex regulation of HIF hydroxylation than was previously derived from enzymatic studies alone. Ratcliffe also made the point that hydroxylase activity is dependent on both the abundance of the PHDs, as well as the cellular availability of oxygen and Fe(II). In addition, the regulation of the HIF hydroxylases is dependent on cell type, and on the transformation state of the tissue, and can be influenced by transcriptional and posttranscriptional events. The potential to stabilize HIF- $1 \alpha$ through small molecules and protect against ischemic injury was presented by Steve Klauss (Fibrogen).

Using an siRNA approach, Jacques Pouyssegur (Centre National de la Recherche Scientifique) demonstrated that PHD2 is the critical oxygen-sensing hydroxylase under normoxic conditions (Berra et al. 2003). Silencing of PHD2 acutely is sufficient to stabilize HIF- $1 \alpha$ in a variety of human cells. PHD2 is a cytoplasmic hydroxylase that is able to shuttle between the cytoplasm and nucleus, and is induced at the transcriptional level by HIF, suggesting an autoregulatory pathway that maintains tight control over HIF protein levels. However, silencing of PHD2 for prolonged periods of time is not sufficient to maintain HIF protein levels, and only when PHD1 is also silenced do HIF levels remain elevated. Interestingly, PHD1 also seems to be activated by a HIFdependent process. Functional analysis of the role of PHDs in development was reported by Peter Carmeliet (University of Leuven). PHD1 and PHD3 homozygous null mice were born at expected or close to expected Mendelian ratios and were fertile. In contrast, PHD2 homozygous null mice died between days 11 and 14 and exhibited severe abnormalities in their placenta. Thus, the severe phenotype of $P H D 2$ null mice is not compensated by PHD 1 and PHD3 and is consistent with it playing an essential role in HIF regulation. However, additional studies will be required to demonstrate that it is the deregulation of HIF that is responsible for the placenta demise and embryonic lethality.

Whereas a great deal of focus has been directed at understanding the role of oxygen sensing in HIF stabilization and its oxygen-degradation domains, the transactivation activity of HIF is also regulated by hydroxylation. Although not involved in the stabilization of HIF-1 $\alpha$, the C-terminal transactivation domain (C-TAD) is involved in modulating transcriptional activation of HIF-1 $\alpha$. Under hypoxic conditions, the C-TAD is able to interact with transcriptional coactivators such as p300/CBP (Ema et al. 1999). However, this interaction requires the inhibition of another oxygen-dependent hydroxylation event, that is, the hydroxylation of the asparagine residue in the conserved domain, YDCEVNV/AP, within the C-TAD (Lando et al. 2002b). Recently, the gene that is responsible for the hydroxylation of asparagine has been identified as the FIH gene (Mahon et al. 2001; Hewitson et al. 2002; Lando et al. 2002a; McNeill et al. 2002). HIF$1 \alpha$ also possesses a $\mathrm{N}$-terminal transactivation domain (N-TAD) that is located in the same region as the oxygen-degradation domain, and C-TAD. The ability of the $\mathrm{C}-\mathrm{TAD}$ to interact with the $\mathrm{CH}-1$ (cysteine/histidine rich) domain of the transcriptional coactivator p300 is mediated by hydroxylation of asparagines 803 in HIF-1 $\alpha$ (Schofield and Ratcliffe 2004). Using an siRNA approach, Nathalie Mazure (Centre National de la Recherche Scientifique) investigated the effect of inhibiting the asparagines hydroxylase $F I H$ alone or in combination with inhibition of PHD2 on HIF transactivation activity. They found that inhibition of FIH alone increased HIF transactivation sixfold, whereas inhibition of $F I H$ as well as PHD2 resulted in a 40-fold increase in HIF transactivation under aerobic conditions, which was similar to that found under hypoxic conditions. Future experiments will be directed at determining the importance of each transactivation domain on HIF target gene expression.

Lorenz Poellinger (Karolinska Institute) identified the two transactivation domains localized in the $\mathrm{C}$ terminus of HIF- $1 \alpha$ as targets of regulation by the transcriptional coactivator SRC-1. These two functional domains of HIF- $1 \alpha$ are contained within 54- or 38-residue-long stretches of amino acids. These same regions of HIF- $1 \alpha$ are also targeted for regulation by other coactivators such as CBP, Ref-1, and, most notably, the combination of Ref-1 together with SRC-1 (Carrero et al. 2000) and $\mathrm{CBP}$, suggesting a link in their mechanisms of action. SRC-1 and CBP constitutively interact with one another, and both proteins have been demonstrated to potentiate steroid hormone receptor-mediated transactivation as a complex, and possesses histone acetyl transferase (HAT) activity (Bannister and Kouzarides 1996; Spencer et al. 1997). Poellinger observed partial reduction of HIF-1 $\alpha$ mediated transcriptional activation with a deletion mutant of $p 300$ that lacks HAT activity. These studies raise the question of whether the transactivation domain of HIF-1 $\alpha$ preferentially interacts with any specific component of the CBP-SRC-1 complex or not, as in vitro studies do not seem to demonstrate that a specific interaction exists. It will also be important to determine whether there are other cofactors that are needed in regulating HIF transactivation.

Interestingly, two additional members of the HIF- $1 \alpha$ family, designated HIF-2 $\alpha$ (also called EPAS1 or MOP2; Ema et al. 1997; Hogenesch et al. 1997; Tian et al. 1997), and HIF- $3 \alpha$ have been identified (Gu et al. 1998). HIF- $2 \alpha$ is highly similar to HIF-1 $\alpha$ in both structure and function, but exhibits more restricted tissue-specific expression, and may as well be differentially regulated by nuclear translocation (Park et al. 2003). This latter point was addressed by Daniel Peet (Adelaide University), who 
demonstrated that the IKK $\gamma$ protein, involved in NF-к $\beta$ signaling, specifically interacts with and regulates HIF$2 \alpha$, but not HIF- $1 \alpha$, under normoxic conditions, and acts to transport HIF- $2 \alpha$ into the nucleus. Peet also stated that whereas recent work has suggested that there are differences in HIF-1 and HIF-2 target genes (Hu et al. 2003), additional studies will be required to determine the cell-type differences in the target genes regulated by these two family members, as well as their differences in their regulation by FIH. Whereas $>40 \mathrm{HIF}$ target genes have been previously described to date, (Semenza 2003) Gregg Semenza (Johns Hopkins School of Medicine) showed that a recent gene expression analysis demonstrated that $\sim 200$ genes were induced by HIF and another 250 genes repressed by HIF. The role of HIF in gene repression is virtually unexplored, and can likely occur by direct binding of HIF to target genes or indirectly through the induction of transrepressor genes such as Dec1 (Yun et al. 2002). In contrast to HIF- $2 \alpha$, HIF-3 $\alpha$ also exhibits conservation with HIF- $1 \alpha$ and HIF- $2 \alpha$ in the $\mathrm{HLH}$ and PAS domains, and an oxygen-degradation domain, but does not possess a similar hypoxia-inducible transactivation domain (Gu et al. 1998).

\section{Ion channels as physiological sensors of hypoxia}

Stephen Archer (University of Alberta) suggests that the rapid response to physiological hypoxia in the ductus arteriosus (DA) is mediated by a sensing unit that is evolutionarily conserved. Closure of the DA is signaled by an oxygen-sensitive vasoconstrictor mechanism in the smooth muscle of the DA (Archer et al. 2004). He proposed that the rapid, vascular oxygen-dependent response is mediated by the oxygen-dependent release of a redox mediator such as $\mathrm{H}_{2} \mathrm{O}_{2}$ by the mitochondria. The release of $\mathrm{H}_{2} \mathrm{O}_{2}$ then alters vascular tone by activating $\mathrm{K}+$ channels in vascular smooth muscle cells. Under hypoxic conditions, the $\mathrm{Kv}$ current in the smooth muscle cells of the pulmonary artery is inhibited, whereas $\mathrm{K}+$ channels are activated in the smooth muscle cell of the ductus arteriosus. It is unclear why there is differential regulation of $\mathrm{K}+$ channels in smooth muscle cells from these different areas; it may relate to differences in the expression of $\alpha$ or $\beta \mathrm{K}+$ channel subunits. This model of $\mathrm{O}_{2}$-sensing may offer a therapeutic target to treat a common respiratory problem associated with preterm infants.

Similar to the changes found in the DA, the glomus cells of the carotid body respond to reduced oxygen by inhibition of $\mathrm{K}+$ channel activity, membrane depolarization, and transmitter release, as discussed by Jose LopezBarneo (Hospital Universitario Virgen del Rocio; LopezBarneo et al. 2004). These transmitters activate the afferent fibers of the sinus nerve that signal the brainstem respiratory center to induce hyperventilation during hypoxia. The critical component of this "membrane model" of oxygen sensing is the potassium channel, that is, voltage-dependent channels $\mathrm{Kv}$, Ca2+-activated $\mathrm{K}+$ channels, and the two pore domain TASK-like $\mathrm{K}+$ channels. However, experimental data has suggested that oxygen tension is detected by some type of oxygen sensor molecule that is located near the ion channel, and not directly by the ion channel itself. For example, oxygen-sensitive $\mathrm{K}+$ channels of rat glomus cells respond to decreased oxygen changes independently of redox modification, (Lopez-Barneo et al. 2004) and the reduction of $\mathrm{K}+$ currents by hypoxia is maintained in airway chemoreceptor cells devoid of mitochondria or after mitochondrial inhibition (Lopez-Barneo et al. 2004). The hypoxia responsiveness of intact glomus cells is unaffected by the complete blockade of the mitochondrial electron flow with saturating concentrations of electron transport chain inhibitors that act on different mitochondrial complexes. However, the Type I mitochondrial complex inhibitor rotenone selectively inhibits the responsiveness of $\mathrm{K}+$ channels to hypoxia (Lopez-Barneo et al. 2004). This suggests that a rotenone-inhibited molecule is essential for carotid body $\mathrm{O}_{2}$ sensing, and that oxygen sensing in the $\mathrm{K}+$ channel may be independent of mitochondrial electron flow. Finally, it appears that ion channels are also regulated by the HIF signaling pathway, and therefore, play a role in the acute and long-term responses to hypoxia.

Nanduri Prabhakar (Case Western Reserve University) discussed the role of carotid bodies as "sensory receptors" for monitoring changes in arterial blood oxygen. He stated that whereas a wide variety of sensors have been proposed to sense oxygen in the carotid body, the question still remains whether the signal transduction involves a single sensor or multiple sensors. He suggested that the putative oxygen sensors in the carotid body work synergistically like a "chemosome", depending on the severity and duration of hypoxic exposure. To test this hypothesis, he exposed rats to either multiple exposures of short durations of hypoxia, or a single exposure to a longer duration of hypoxia (Peng and Prabhakar 2004). He found that the hypoxic sensory response of the carotid body was significantly enhanced in animals treated with short durations of hypoxia, but not in animals treated for a single long-term treatment. This effect was evident if the carotid body was treated ex vivo. The hypoxic sensory response of the carotid body was reversible after the animal was returned to a normoxic environment. If the short-duration treated animals were also treated with an $\mathrm{O}_{2}$ radical scavenger, the hypoxic sensory response of the carotid was greatly diminished, suggesting that superoxide radicals play an important role in carotid body sensing of hypoxia, but not necessarily through potassium channel activity.

\section{Oxygen sensing and development}

\section{Model organisms}

Many organs, including the mammalian lung and the cardiovascular system, consist of branched tubular networks that transport essential gases or fluids; but, the genetic programs that control the development of these complex three-dimensional structures are not well understood. Mark Krasnow (Stanford University) described 
the Drosophila melanogaster tracheal system used for respiration in insects as a network of interconnected epithelial tubes that transport oxygen and other gases through the body; he discussed how they provide a unique model to study the effect of hypoxia on branching morphogenesis. There are both hard-wired signals, as well as environmental signals that control this development (Ghabrial et al. 2003). The primary and secondary branches of the trachea are not influenced by changes in oxygen, whereas the development of the tertiary branches is oxygen sensitive. Branching in the respiratory system is controlled by fibroblast growth factor (FGF) and fibroblast growth factor receptor (FGFR). During terminal branching, FGF expression is regulated by hypoxia, ensuring that tracheal structure matches cellular oxygen need. The signal-transduction pathway that regulates FGF gene expression in Drosophila is controlled by the inhibition of prolyl hydroxylation and activation of the Drosophila homolog of HIF, Sima, in an analogous manner to that found in mammalian cells. Model systems like Drosophila allow genetic screens to understand complex developmental phenotypes, and one such screen done by Krasnow's group showed the genetic basis of a tortuous trachea phenotype, including identification of the gene involved, Tortuous trachea-1. This raises the possibility that the tortuous and malformed vasculature that is often found in solid tumors, which has been thought to arise from dysregulated angiogenic growth-factor production, could, in fact, be the result of the mammalian homologs for genes such as Tortuous trachea-1. Further research on homologous genes in mammals should prove very interesting in this regard.

Frank Bunn (Brigham and Women's Hospital) reported that in Drosophila SL2 cells, the Drosophila HIF homo$\log$ Sima is found as a full-length transcript and as a splice variant (Gorr et al. 2004). The full-length Sima isoform is regulated by hypoxia, iron chelation, and transition metals in a manner similar to mammalian HIF- $1 \alpha$. However, the splice variant lacks an oxygen-degradation domain and nuclear localization sequence, and can act to regulate Sima function by binding to the Drosophila ARNT (HIF-1 $\beta$ ) homolog Tango in the cytoplasm. Lorenz Poellinger and colleagues have previously reported that IPAS, a 307 amino acid protein that contains a bHLH PAS motif, is predominantly expressed in Purkinje cells of the cerebellum in mice, as well as in corneal epithelium of the eye, where it appears to play a role as a negative regulator of angiogenesis and maintenance of an avascular phenotype (Makino et al. 2001). Sequencing of the mouse IPAS genomic structure revealed that IPAS is a splicing variant of the HIF- $3 \alpha$ locus. Thus, mammalian cells seem to possess a mechanism for regulating HIF-1 that is similar to Drosophila.

Chris Bradfield (University of Wisconsin) discussed a more general role of the PAS proteins AHR (aromatic hydrocarbon receptor) and ARNT (HIF-1 $\beta$ ) as environmental sensors of planar aromatic compounds, oxygen sensing, and light sensing (Gu et al. 2000). The physiological role for these genes has come from gene-target- ing experiments in mice, where mouse strains deficient in the AHR protein show defects in liver development, decreased animal weights, and poor fecundity $(\mathrm{Gu}$ et al. 2000). As predicted, $A H R$ null mice fail to induce xenobiotic metabolizing enzymes in response to exposure to dioxins ( $\mathrm{Gu}$ et al. 2000). Murine strains lacking the ARNT protein display embryonic lethality between 9.5 and $10.5 \mathrm{~d}$ of gestation and defective angiogenesis (Maltepe et al. 1997). The generation of mice with hypomorphic alleles of $A R N T$ has shown that they react like wild-type mice in response to dioxin challenge, but still are unable to close their ductus venosus after birth, indicating that these mice will be useful in dissecting the role of ARNT in the AHR and HIF pathways. Taken together, these data support the concept that the ability to respond to changes in oxygen is essential for development.

Randy Johnson (University of San Diego) discussed the phenotype of HIF-1 $\alpha$-deficient animals and the role of HIF-1 heterodimer in the hypoxia-induced transcriptional activation of genes involved in developmental angiogenesis and glycolysis (Semenza 2003). However, the phenotype of HIF-2 $\alpha$ null mice provided a surprising result. Previous studies had suggested that $H I F-2 \alpha^{-/}$embryos die at day 12.5 of gestation due to pronounced bradycardia related to substantially decreased catecholamine levels; although at least one other group found that the loss of viability was instead related to defective vascularization (Tian et al. 1998; Peng et al. 2000; Compernolle et al. 2002). Joe Garcia (University of Texas Southwestern Medical Center) used the mixed $129 \times \mathrm{B} 6$ strain of mice and obtained viable HIF-2-deficient animals. These animals exhibited reduced lifespan, sterility, retinopathy, decreased hematopoietic lineages, and increased heart and liver size. Insight into the defects in these mice came from the observation that they possess systemic lipid droplets in skeletal muscle, heart, and liver, due to impaired fatty acid oxidation /Scortegagna et al. 2003). The decreased utilization of free fatty acids and lower respiratory control in the mitochondria from these mice exhibited striking similarities to SOD2 (MnSOD)deficient mice. Support of this concept came from treating mice with the SOD mimetic MnTBAP, which reversed the increased liver deposits found in these mice. In addition, pregnant HIF- $2 \alpha$-deficient mice treated with MnTBAP exhibited decreased embryonic lethality. Future studies will be needed to understand the unexpected role of superoxide in HIF-2 $\alpha$-deficient mice.

Volker Haase (University of Pennsylvania) presented data on the importance of pVHL on normal development and tumor formation. He found that mice with a homozygous pVHL deficiency die during midgestation from hypovascularization of the placenta (Haase et al. 2001). Therefore, he developed a Cre-loxP system to generate conditional floxed $V H L$ alleles. The only manifestation of haplosufficient VHL mice is that they develop cavernous hemangiomas at an old age. These hemangiomas in $\mathrm{VHL}^{+/-}$mice result from dysregulation of HIF in hepatocytes and not endothelial cells. Crossing the floxed $V H L$ with the PEPCK-CRE that allows kidney-specific CRE 
expression in the mouse resulted in systemic erythrocytosis, some tubular atrophy, single cysts, but no dysplastic lesions. The double $V H L$ and $A R N T$ conditional knockout mice resulted in a less-severe phenotype in liver. However, the double VHL and HIF-1 $\alpha$ conditional mice did not rescue the phenotype, implying that HIF-2 and not HIF-1 is involved in the formation of the observed vascular lesions. Therefore, ARNT loss prevents VHL-associated hepatic vascular lesion development, but HIF-1 $\alpha$ loss is not sufficient. Somewhat surprisingly, proximal renal tubule cells in PEPCK-CRE VHL knockout mouse did not exhibit dysplastic lesions and did not appear to be a source of increased erythropoietin (EPO) production. Thus, the question remains as to what is the cell type in the kidney in which mutations in pVHL result in dysplastic lesions and renal-cell carcinoma? Volker Haase also presented data to indicate that VHL deficiency resulted in small hypervascular thymi. In particular, CD4/CD8 double-positive thymocytes were significantly reduced in number due to increased apoptotic cell death. This death was rescued by HIF-1 $\alpha$ deficiency, but not by Bcl-2 loss. Loss of pVHL in intestinal epithelial cells using a fatty acid-binding protein cre promoter mouse cross resulted in protection against TNBS colitis that is pathologically known to be associated with inflammation and tissue hypoxia by increasing epithelial barrier function. In collaboration with Celeste Simon, experiments showed that loss of the VHL tumor-suppressor gene in embryonic stem cells or in fibrosarcomas did not promote teratocarcinoma growth (Mack et al. 2003). One explanation for this finding lies in the fact that the cell cycle inhibitor p27 is elevated. Thus, why loss of VHL promotes malignant progression in renal cells, but not in other cell types, requires further investigation.

\section{Adaptation to hypoxia}

One of the first hypoxia and HIF-regulated genes identified was the EPO gene. Whereas increased hematocrit is associated with hypertension and thromboembolism, individuals who live at high altitudes compensate for increased erythrocyte levels. Max Gassman (University of Zurich) described the generation of transgenic mice that overexpressed EPO in a hypoxia-independent manner and that possessed hematocrits between 0.8 and 0.9 , nearly double that found in wild-type mice (Vogel et al. 2003). Unexpectedly, these mice did not exhibit increased blood pressure, heart rate, or cardiac output, although they exhibited splenomegaly. The adaptation of these transgenic mice to high hematocrit levels is the result of regulated elevation of blood viscosity by increasing erythrocyte flexibility. Interestingly, overexpression of EPO protected mice from myocardial infarction and from light-induced retinal degeneration. EPO transgenic mice also swim and run longer, due to increased capillary density in their skeletal muscle, but possess decreased neuromuscular junctions. This hypoxia-independent EPO transgenic mouse model has been instructive in understanding the physiological consequences of adaptation to high EPO levels.

Jeff Arbeit (Washington University) investigated the ability of skin to adapt to the overexpression of a HIF-1 $\alpha$ gene that lacks an oxygen-degradation domain (ODD). The purpose of these experiments was to understand the effects of a HIF-1 $\alpha$ gain-of-function in differentiating epi-

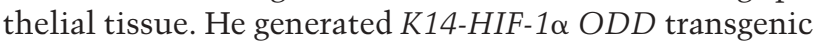
mice that expressed HIF constitutively in their basal keratinocytes (Elson et al. 2001). Although the mice exhibited redness of unfurred skin due to an increase in microvessels, no apparent phenotype was discernable in the skin, despite overexpression of HIF-1 target genes such as VEGF. In fact, the skin of the K14-HIF-1 $\alpha$ ODD mice, as well as the corneas, were found to be leakage resistant, due to a failure to prune neonatal vasculature associated with persistent elevation of VEGF mRNA. These results suggest that constitutive expression of HIF-1 $\alpha$ in keratinocytes results in hypervascularity that is not progressive into angiomas. Interestingly, treatment of these same transgenic mice with DMBA/TPA resulted in reduced size and number of papillomas that formed. However, after 16-27 wk, the papillomas that formed became more invasive, suggesting that HIF-1 is a late-stage progression factor in skin cancer.

The seminiferous tubules of the testes are poorly vascularized and are under low-oxygen tension. Roland Wenger (University of Zurich-Irchel) described a novel $H I F-1 \alpha$ isoform, termed hHIF- $1 \alpha \mathrm{Te}$, which is exclusively expressed in human testis (Depping et al. 2004). The predicted coding region lacks 59 amino acids of the N-terminal bHLH domain, resulting in a dominant-negative function of hHIF-1 $\alpha$. He proposed that the testis-specific expression of a dominant-negative regulator of normal HIF-1 function might be involved in the inhibition of a subset of genes transcriptionally regulated by HIF-1. Because testis-specific variants of many of the glycolytic enzymes are expressed exclusively during spermatogenesis, inhibition of normal HIF-1 function might contribute to the decrease in expression of the ubiquitous isoforms of the glycolytic enzymes, which are all under the transcriptional control of HIF-1. Inhibition of transcriptional activity by dominant-negative regulation is a common mechanism in testis (e.g., the Id protein). Roland Wenger also discussed a novel, mammalian PAS domain protein that is highly expressed in the testis, PASKIN (Katschinski et al. 2003). However, it is unclear what physiological role it plays in the testes.

Stella Kourembanas (Harvard Medical School) presented evidence that hypoxia stimulates vasoconstriction and vessel-wall remodeling of the pulmonary vasculature; these changes are contributors to pulmonary hypertension. Hypoxia increases the proliferative response of vascular smooth muscle cells to mitogens, and prolongs their lifespan by increasing telomerase activity (Minamino and Kourembanas 2001). Hypoxia also induces inflammatory cell infiltration in the lung, in part through the transcriptional induction of macrophage inflammatory protein by NF-кB. Lung adaptation to hypoxia is through the induction of the heme oxygenase 
gene (HO-1), whose product generates the antioxidant bilirubin and the vasodilator carbon monoxide (Minamino et al. 2001). Kourembanas' studies showed that cell lines and mice genetically modified to produce HO-1 demonstrated reduced proliferation and inflammation when challenged by hypoxia. These studies have important implications for the pharmacologic manipulation of HO-1 to allow lung tissue to adapt to hypoxia.

Larissa Shimoda (Johns Hopkins School of Medicine) investigated the role of ion homeostasis in pulmonary arterial pressure during contraction and proliferation of pulmonary vascular smooth muscle in rodents exposed to $10 \%$ oxygen for 3 wk (Raj and Shimoda 2002). She found that pulmonary smooth muscle cells from these animals display attenuated voltage gated $\mathrm{K}+(\mathrm{Kv})$ channel activity, depolarization of the membrane potential, elevated resting intracellular $\mathrm{Ca} 2+$, and an alkaline shift in intracellular $\mathrm{pH}$. The inhibition of the Kv channel activity and membrane depolarization is the result of decreased $\mathrm{Kv}$ channel expression, whereas the alkaline shift in $\mathrm{pH}$ is the consequence of increased $\mathrm{Na}+\mathrm{H}+$ exchanger expression and activity.

Ivan McMurtry (University of Colorado Health Sciences Center) discussed the role of the small GTP-binding protein RhoA and its downstream effector Rho kinase in mediating sustained pulmonary vasoconstriction in pulmonary hypertension (McMurtry et al. 2003). He found that treatment of rats that had been exposed to 3 wk of hypobaric oxygen (10\% oxygen) with the Rho kinase inhibitor Y-27632 reversed pulmonary vascular resistance and completely reversed vasoconstriction elicited by inhibition of NO synthesis. If rats were exposed to hypoxia while being simultaneously treated with Y-27632, the severity of pulmonary hypertension, vascular remodeling, and right ventricular hypertrophy were reduced. However, systemic arterial pressure was unchanged. These studies suggest that Rho kinase signaling also contributes to pulmonary hypertension along with changes in ion channel homeostasis, and that inhibition of Rho kinase signaling can, in part, ameliorate the consequences of pulmonary hypertension induced by hypoxia.

Frank Giordano (Yale University) investigated the role of HIF-1 in adaptive changes in oxygen delivery to the myocardium. Using cardiac specific deletion of HIF-1 $\alpha$, he found that the mice were viable, but have cardiac dysfunction at basal normoxic conditions, and reduced cardiac vascularity (Huang et al. 2004). Furthermore, the rate of developed pressure and relaxation were substantially reduced in $H I F-1 \alpha$-deficient hearts when compared with wild-type counterparts. These findings correlated with reduced contractility at the cardiac myocyte level and prolongation of calcium transients. Metabolically, $H I F-1 \alpha$-deficient hearts generated less ATP under normoxic conditions and exhibited reduced expression of numerous HIF-1 target genes. In contrast to HIF- $1 \alpha$ deficient hearts, deletion of $V H L$ in the heart resulted in $100 \%$ mortality by 5 mo of age, which was associated with hypervascularity and myocyte loss and replacement fibrosis. The increased fibrosis correlated with in- creased levels of TGF $\beta 1$. These results demonstrate the importance of regulated HIF-1 expression in cardiac myocytes under normoxic conditions.

Kenneth Walsh (Boston University) explored the protective role of Akt using a transgenic mouse system in which Akt could be conditionally activated in cardiac myocytes (Fujio et al. 2000; Miao et al. 2000). He found that short-term activation of Akt resulted in reversible hypertrophy and cardioprotection when the heart was challenged by stress. However, prolonged Akt expression induced severe cardiac hypertrophy and ventricular remodeling. Thus, in considering genetic or pharmacological approaches to use Akt for treatment of heart disease, only short-term treatments may prove beneficial, with chronic treatment, leading to heart failure.

Using a hypoxia-specific marker, Ernestina Schipani (Massachusetts General Hospital) showed evidence that the fetal chondrocytic growth plate (central region) contains a hypoxic region, and also stains positive for HIF- $1 \alpha$ (Schipani et al. 2001). Loss of HIF-1 $\alpha$ expression in chondrocytes results in shortened limbs and a malformed sternum with no clear cellular element. Loss of HIF-1 $\alpha$ causes increased cell death in the chondrocytic growth plate. Cells that lack HIF-1 $\alpha$ in the interior of the growth plate die by apoptosis and fail to undergo growth arrest. VEGF expression in the growth plate is regulated through both HIF- $1 \alpha$-dependent and HIF- $1 \alpha$-independent mechanisms. Surprisingly, VEGF expression is elevated in a HIF- $1 \alpha$-independent manner in chondrocytes surrounding areas of cell death, and this, in turn, induces ectopic angiogenesis. HIF-1 $\alpha$-null chondrocytes are also unable to maintain ATP levels in hypoxic microenvironments, indicating a fundamental requirement for this factor in the regulation of chondrocyte metabolism. Thus, HIF plays multiple roles in chondrocyte development by providing growth arrest and survival signals that allow chondroblasts to adapt and differentiate in a hypoxic environment.

Mammalian lenses are avascular throughout most of life, but continue to grow in a hypoxic environment, indicating that they have adapted to a low-oxygen environment. David Beebe (Washington University) presented results from experiments, in which he investigated the role of oxygen in regulating lens growth in vivo. He found that young rats exposed to $60 \%$ oxygen compared with normoxic conditions exhibited little change in proliferation as assayed by BrdU labeling. In older rats, BrdU labeling was low under normoxic conditions, but increased to the same level as the younger rats upon exposure to hypoxia. At the cellular level, the rate of lens-fiber cell production also increased in rats exposed to $60 \%$ oxygen. Again, HIF- 1 seems to play an important role in lens development and adaptation to hypoxia, as HIF-1 $\alpha$-deficient lens showed severe disruption of epithelial and fiber cell morphology and degenerated postnatally. In younger rats, hypoxia has little effect on epithelial proliferation or lens growth. In older rats, hypoxia is essential in inhibiting epithelial proliferation and lens growth. How HIF inhibits proliferation in the aging lens could provide insight into the tissue-specific 
effects of HIF on growth regulation and adaptation to a low-oxygen environment.

Gregg Semenza discussed the importance of physiologic responses to hypoxia that are mediated by the transcriptional activator HIF-1 and its downstream targets (Semenza 2004). He gave a variety of examples of the importance of HIF1 cardiorespiratory physiology. For example, in mice with loss of one HIF-2 $\alpha$ allele, hypoxiainduced expression of endothelin-1 and norepinephrine is significantly reduced, and these mice fail to develop pulmonary hypertension after four weeks of exposure to $10 \%$ oxygen. In mice with loss of one HIF-1 $\alpha$ allele, the carotid body is unable to properly signal a response to reduced oxygen conditions. In mice with loss of one HIF$1 \alpha$ allele, intermittent hypoxia does not induce EPO production or cardiac protection when compared with wildtype mice with two functioning HIF alleles. He also presented experimental data that EPO provides potent protection against ischemia-reperfusion injury in the heart. These results further support an important, but yet complex role for HIF in maintaining normal tissue homeostasis in response to changing oxygen conditions.

\section{Hypoxia, HIF, and oncogenesis}

A major question regarding HIF is its role in oncogenesis. Adrian Harris (Oxford University) reported that head and neck cancer patients negative for HIF- $2 \alpha$ and carbonic anhydrase IX (CAIX) had the best prognosis from the analysis of the European CHART trials, suggesting that the combination of the two molecular markers may be useful for prognosis in the future (Potter and Harris 2004). Amato Giaccia (Stanford University) reported that HIF itself did not act as a classical oncogene, in that it could not transform NIH 3T3 cells or melanocytes in vitro. However, overexpression of an ODD-deficient HIF-1 increased transformation and tumor growth of mouse cells. To date, no gain-of-function mutations in HIF that make it stabilized under aerobic conditions or increase its transactivation potential have been identified in tumor cells. Gregg Semenza presented additional evidence that overexpression of HIF-1 $\alpha$ enhanced the growth of transplanted tumors in immune-deficient mice, and that inhibition of HIF through the use of dominant-negative HIF inhibited tumor growth (Semenza 2003). Frank Lee (University of Pennsylvania) reported that proline 582 to serine polymorphism that is found at a frequency of 0.073 in the population did not affect HIF hydroxylation or transactivation. Whereas HIF itself does not seem to be mutated in pathological disorders or neoplastic cells, a variety of other factors that affect HIF stability are dysregulated or inactivated. Cell lines derived from tumors that have lost the VHL tumor-suppressor gene display aerobic HIF- $1 \alpha$ protein expression (Maxwell et al. 1999; Ohh et al. 2000). Tumors such as renal cell carcinomas (RCC) that possess mutations in VHL also exhibit high aerobic expression of HIF-1-regulated genes, whereas reintroduction of wild-type $V H L$ substantially reduces the aerobic level of HIF- $1 \alpha$ protein to those found in untransformed or transformed cells that express wild-type VHL. Bill Kaelin (Dana-Farber Cancer Institute) presented data that indicates that a HIF- $2 \alpha$ variant lacking both of its two prolyl hydroxylation/pVHL-binding sites prevents tumor inhibition by $\mathrm{pVHL}$ in a DNA-binding-dependent manner (Kondo et al. 2003). Furthermore, inhibition of HIF-2 $\alpha$ expression with short hairpin RNAs is sufficient to suppress tumor formation by $\mathrm{pVHL}$-defective renal carcinoma cells (Kondo et al. 2003). These results strongly argue that tumor suppression by pVHL is tightly associated with the regulation of HIF protein.

Other pathways were discussed by Bill Kaelin that impact HIF activity by affecting TOR activity. For example, loss of the tuberous sclerosis complex tumor-suppressor gene (TSC-2) results in hamartomas. Mutant versions of TSC-2 have little effect on mTOR and HIF accumulation, whereas wild-type TSC-2 inhibits mTOR and HIF accumulation (Brugarolas et al. 2003). Wayne Zundel (University of Colorado Health Sciences Center) reported that CSN5 subunit of the COP9 signalsome holocomplex interacts directly with both the CODD (independent of prolyl-hydroxylation state) and the pVHL E3 ligase. He concluded that CSN5, pVHL, and the PHDs interact with the same region of HIF- $1 \alpha$, and therefore, CSN5 alters pVHL or PHD affinity for HIF-1 $\alpha$ under aerobic conditions (Bemis et al. 2004).

\section{Exploiting tumor hypoxia}

Over the last decade, numerous preclinical and clinical studies have indicated that hypoxia is an important indicator of therapeutic response to radiotherapy, chemotherapy, and even surgery. It decreases the effectiveness of radiation that requires free radicals to kill cells, makes them refractory to killing by chemotherapy agents that require rapidly proliferating cells to be effective, and selects for tumor cells that are highly aggressive. Peter Vaupel (University of Mainz) discussed the mechanisms leading to tumor hypoxia (Vaupel 2004). He stated that there are three major mechanisms that cause tumor hypoxia: (1) the inability of the structurally abnormal vessels in the tumor to perfuse tumor cells adequately; (2) interrupted microcirculation, and (3) tumor or therapyinduced anemia. Additional contributory mechanisms include carboxyhemoglobin ( $\mathrm{HbCO}$ ) formation in heavy smokers and hypoxemia in microvessels arising from the venous side of the vascular network. In the new age of targeted therapy, pretreatment assessment of hypoxia in patients with solid tumors could allow them to benefit from the addition of a hypoxia directed therapeutic.

Currently, a variety of strategies exist to exploit tumor hypoxia for treatment. Dietmar Siemann (University of Florida) discussed a number of these strategies; they include overcoming tumor hypoxia through the administration of agents such as EPO, exploiting tumor hypoxia through the use of bioreductive agents that are activated under hypoxic conditions, and increasing tumor ischemia through the use of vascular targeting agents. $\mathrm{He}$ discussed treating malignant disease by targeting the blood vessel support networks of tumors, a topic that has 
received a great deal of attention in recent years, albeit more from the antiangiogenic side than from the vascular targeting side. Vascular targeting agents differ from anti-angiogenic agents, in that they destroy established vasculature (Siemann et al. 2004). Preclinical studies have demonstrated that vascular targeting agents can produce rapid and significant vascular effects that, once initiated, lead to widespread, dose-dependent tumor necrosis in a matter of hours or days. Whereas these agents seem to be highly effective in reducing tumor size, they alone do not eradicate tumors, as tumor cells that can obtain their oxygen and nutrients by diffusion could survive and repopulate the tumor. Therefore, a vascular targeting agent should be combined with radiotherapy or chemotherapy to improve tumor response. In early Phase I and Phase II clinical trials, vascular targeting agents have exhibited the ability to produce significant reductions in tumor blood flow at acceptable doses. The future for these agents looks promising.

Martin Brown (Stanford University) described one mechanism by which a nontoxic prodrug can be activated to a toxic drug under hypoxic conditions, as a model for hypoxia specific cytotoxins. (Brown and Wilson 2004) He stated that molecules that can undergo one-electron reduction to a radical can be reoxidized to the original compound under normoxic conditions, but will not be reoxidized under hypoxic conditions, and thus will retain their ability to induce DNA damage. One example of a drug that is activated under hypoxic conditions is Tirapazamine (TPZ). TPZ potentiates the antitumor effect of radiation by selectively killing the radiation-resistant hypoxic cells in tumors. It also enhances killing of hypoxic tumor cells treated with cisplatin. Whereas a Phase-III study with cisplatin-based chemoradiotherapy is now underway, the dose of TPZ that can be administered during chemoradiation is limited by the side effects of neutropenia. Thus, whereas the concept has been demonstrated to work, new hypoxiaactivated prodrugs are needed. Martin Brown also discussed clostridial-dependent enzyme prodrug therapy (CDEPT). This approach relies on the spore form of clostridia germinating in the hypoxic/necrotic regions of solid tumors and releasing the prodrug-activating enzyme in areas of the tumor that have hypoxic tumor cells and far from blood vessels. Obviously, there would be a potential advantage to combine vascular targeting therapy that would increase tumor necrosis and hypoxic with CDEPT.

Hypoxia-targeted therapies such as clostridia or TPZ act to kill hypoxic cells in a nonselective manner. Giovanni Melillo (National Cancer Institute) described a recent screen of 2000 compounds that represent a "Diversity Set" of the NCI's repository to identify HIF inhibitors (Rapisarda et al. 2002). This screen was instrumental in demonstrating that pharmacological inhibitors of HIF$1 \alpha$ could be screened in a high-throughput fashion, and attempted to exclude compounds that were cytotoxic, on the basis of the changes in expression of a second, constitutively expressed luciferase reporter gene. One group of compounds that Melillo and colleagues found had ac- tivity against HIF-1 $\alpha$, and also topoisomerase I. This group is exemplified by the compound NSC-609699 (topotecan; Rapisarda et al. 2002). Topotecan does not affect HIF- $1 \alpha$ protein accumulation, but inhibits its translation. He also presented data that indicates that Topoisomerase 1 is required for the inhibition of HIF-1 $\alpha$ protein accumulation by topotecan; camptothecin-resistant cell lines with known Topo 1 alterations do not exhibit HIF-1 $\alpha$ inhibition when treated with topotecan (Rapisarda et al. 2004). He also showed that topotecan possessed anti-tumor activity against transplanted tumors in immunodeficient mice. A clinical trial is being planned to test the hypothesis that HIF-1 inhibition in tumors will result in increased tumor control and survival.

Amato Giaccia stated that, whereas the effect of loss of HIF-1 $\alpha$ in tumors leads to inhibition of tumor growth, it does not eliminate tumors. Studies indicate that inhibition of HIF-1 leads to a tumor growth delay, but that in some tumors, growth can eventually resume in a HIF-1independent manner. Therefore, it would be therapeutically more beneficial to eliminate cells with elevated HIF than to inhibit their growth (Sutphin et al. 2004). Thus, a HIF-directed cytotoxin could be found that would kill cells when HIF activity is elevated, such as by hypoxia, oncogenes, or growth-factor dysregulation. He reported on the isolation of a drug that kills cells deficient in VHL preferentially and that possess elevated HIF-1 activity. He stated the importance for a large highthroughput screen to find different families of such drugs to test in preclinical models for efficacy.

Mark Dewhirst (Duke University) presented the paradox of HIF-1 signaling during tumor reoxygenation/irradiation treatment. Last year, Garcia-Barros et al. published a paper that indicated that changing the intrinsic radiation sensitivity of the tumor vasculature resulted in increased regression of tumors treated with radiotherapy (Garcia-Barros et al. 2003). Previous studies have suggested a correlation between HIF-1 and an outcome in patients treated with radiotherapy (Aebersold et al. 2001) that could be explained by the fact that increased levels of HIF represented increased tumor hypoxia or dysregulated growth-factor expression. Mark Dewhirst presented data indicating that radiation-treated tumors induce HIF-1, which results in increased VEGF and bFGF that can act as survival factors for endothelial cells (Moeller et al. 2004). Surprisingly, it is radiation-induced reoxygenation of tumors that results in reactive oxygen species that induces HIF-1. Whether these reactive oxygen species differ from those generated by the mitochondria still needs to be determined, as well as their mechanisms of action. Do they increase HIF accumulation through a prolyl hydroxylase inhibition or through another mechanism? He also demonstrated the potential translation of these studies to the clinic by pretreating mice with the free radical scavenger AEOL-10113, which blocks the induction of HIF-1 activity, or YC-1, which is a small-molecule inhibitor of HIF-1, before irradiating them. Treatment with either compound that inhibits HIF resulted in increased tumor growth delay. In vitro 
reoxygenation of tumor cells results in a rapid decay in HIF-1 protein, suggesting that there is something unique about the reoxygenation of tumor cells that results in an increase in HIF and HIF target genes. A recent study by Mekhail may lend some insight into the fact that this increased level of HIF after reoxygenation occurs in regions of the tumor that are at low $\mathrm{pH}$ and result in decreased VHL activity (Mekhail et al. 2004). Thus, these studies suggest that HIF inhibitors would have a dual role in the tumor, in that they inhibit tumor cell growth and increase the sensitivity of endothelial cells to radiotherapy by inhibiting the production of endothelial cells survival factors. It will be important to determine the efficacy of this approach in human tumors. In contrast to treating solid tumors with an intact VHL and HIF pathway, the treatment of patients with pVHL-deficient tumors, such as those found in renal cell carcinoma, is prime for the concept of targeted therapy. Bill Kaelin stated that drugs that inhibit VEGF or its receptor have shown activity in clinical trials against renal cell carcinoma (Rini et al. 2004). As HIF-2 is sufficient and necessary for tumor growth and expansion of pVHLdeficient tumors, then the combination of HIF inhibitors to increase endothelial sensitivity and radiotherapy may have now some efficacy, where it previously had none.

\section{Conclusion}

Hypoxia-inducible responses are highly regulated in normal embryonic development and are dysregulated in a number of disease states, including cancer. The identification of oxygen-regulated proteins, such as the HIF family transcription factors, serves as a paradigm for oxygen sensing at the molecular level, but probably represents only one example on a much larger theme of protein regulation in response to hypoxia. A variety of intracellular organelles have also been implicated in oxygen sensing, such as ion channels, mitochondria, and the endoplasmic reticulum, and it will be important to clearly delineate whether they sense in changes in oxygen tension directly or through as yet unknown mechanisms. The use of model organisms, including transgenic and knockout mice, has been very instructive in linking molecular events in oxygen sensing with developmental responses, and should provide a more complete framework to understand what aspects of oxygen sensing are conserved during evolution. Finally, whereas the role of hypoxia and hypoxia-induced gene expression in malignant progression and response to therapy is supported by numerous studies, the question of the contributions of hypoxic tumor stroma and secretion of cytokines and chemokines to malignant progression is poorly understood and requires further study. The fundamental knowledge of how cells sense and respond to hypoxia has provided an important framework to understand the role of hypoxia in disease and cancer. The goal is that we can ultimately exploit this knowledge for diagnosis and treatment.

\section{Acknowledgments}

We thank the Keystone Society for their help and generosity in organizing this meeting. We would especially like to acknowledge all of the contributions to the field of hypoxia research that were made at this meeting that we were unable to properly acknowledge in this meeting review.

\section{References}

Aebersold, D.M., Burri, P., Beer, K.T., Laissue, J., Djonov, V., Greiner, R.H., and Semenza, G.L. 2001. Expression of hypoxia-inducible factor- $1 \alpha$ : A novel predictive and prognostic parameter in the radiotherapy of oropharyngeal cancer. Cancer Res. 61: 2911-2916.

Archer, S.L., Wu, X.C., Thebaud, B., Moudgil, R., Hashimoto, K., and Michelakis, E.D. 2004. $\mathrm{O}_{2}$ sensing in the human ductus arteriosus: Redox-sensitive $\mathrm{K}+$ channels are regulated by mitochondria-derived hydrogen peroxide. Biol. Chem. 385: 205-216.

Arsham, A.M., Howell, J.J., and Simon, M.C. 2003. A novel hypoxia-inducible factor-independent hypoxic response regulating mammalian target of rapamycin and its targets. $J$. Biol. Chem. 278: 29655-29660.

Bannister, A.J. and Kouzarides, T. 1996. The CBP co-activator is a histone acetyltransferase. Nature 384: 641-643.

Bemis, L., Chan, D.A., Finkielstein, C.V., Qi, L., Sutphin, P.D., Chen, X., Stenmark, K., Giaccia, A.J., and Zundel, W. 2004. Distinct aerobic and hypoxic mechanisms of HIF-a regulation by CSN5. Genes \& Dev. 18: 739-744.

Berra, E., Benizri, E., Ginouves, A., Volmat, V., Roux, D., and Pouyssegur, J. 2003. HIF prolyl-hydroxylase 2 is the key oxygen sensor setting low steady-state levels of HIF-1 $\alpha$ in normoxia. EMBO J. 22: 4082-4090.

Brown, J.M. and Wilson, W.R. 2004. Exploiting tumour hypoxia in cancer treatment. Nat. Rev. Cancer 4: 437-447.

Brugarolas, J.B., Vazquez, F., Reddy, A., Sellers, W.R., and Kaelin Jr., W.G. 2003. TSC2 regulates VEGF through mTOR-dependent and -independent pathways. Cancer Cell 4: 147-158.

Carrero, P., Okamoto, K., Coumailleau, P., O’Brien, S., Tanaka, H., and Poellinger, L. 2000. Redox-regulated recruitment of the transcriptional coactivators CREB-binding protein and SRC-1 to hypoxia-inducible factor $1 \alpha$. Mol. Cell. Biol. 20: 402-415.

Compernolle, V., Brusselmans, K., Acker, T., Hoet, P., Tjwa, M., Beck, H., Plaisance, S., Dor, Y., Keshet, E., Lupu, F., et al. 2002. Loss of HIF- $2 \alpha$ and inhibition of VEGF impair fetal lung maturation, whereas treatment with VEGF prevents fatal respiratory distress in premature mice. Nat. Med. 8: 702-710.

Depping, R., Hagele, S., Wagner, K.F., Wiesner, R.J., Camenisch, G., Wenger, R.H., and Katschinski, D.M. 2004. A dominantnegative isoform of hypoxia-inducible factor- $1 \alpha$ specifically expressed in human testis. Biol. Reprod. 71:331-339.

Elson, D.A., Thurston, G., Huang, L.E., Ginzinger, D.G., McDonald, D.M., Johnson, R.S., and Arbeit, J.M. 2001. Induction of hypervascularity without leakage or inflammation in transgenic mice overexpressing hypoxia-inducible factor- $1 \alpha$. Genes \& Dev. 15: 2520-2532.

Ema, M., Taya, S., Yokotani, N., Sogawa, K., Matsuda, Y., and Fujii-Kuriyama, Y. 1997. A novel bHLH-PAS factor with close sequence similarity to hypoxia-inducible factor $1 \alpha$ regulates the VEGF expression and is potentially involved in lung and vascular development. Proc. Natl. Acad. Sci. 94: 4273-4278

Ema, M., Hirota, K., Mimura, J., Abe, H., Yodoi, J., Sogawa, K., 
Poellinger, L., and Fujii-Kuriyama, Y. 1999. Molecular mechanisms of transcription activation by HLF and HIF $1 \alpha$ in response to hypoxia: Their stabilization and redox signalinduced interaction with $\mathrm{CBP} / \mathrm{p} 300$. EMBO J. 18: 19051914.

Fujio, Y., Nguyen, T., Wencker, D., Kitsis, R.N., and Walsh, K. 2000. Akt promotes survival of cardiomyocytes in vitro and protects against ischemia-reperfusion injury in mouse heart. Circulation 101: 660-667.

Garcia-Barros, M., Paris, F., Cordon-Cardo, C., Lyden, D., Rafii, S., Haimovitz-Friedman, A., Fuks, Z., and Kolesnick, R. 2003. Tumor response to radiotherapy regulated by endothelial cell apoptosis. Science 300: 1155-1159.

Ghabrial, A., Luschnig, S., Metzstein, M.M., and Krasnow, M.A. 2003. Branching morphogenesis of the Drosophila tracheal system. Annu. Rev. Cell Dev. Biol. 19: 623-647.

Gorr, T.A., Tomita, T., Wappner, P., and Bunn, H.F. 2004. Regulation of Drosophila HIF activity in SL2 cells: Identification of a hypoxia-induced variant isoform of the HIFa homologue gene similar. J. Biol. Chem. (in press).

Gu, Y.Z., Moran, S.M., Hogenesch, J.B., Wartman, L., and Bradfield, C.A. 1998. Molecular characterization and chromosomal localization of a third $\alpha$-class hypoxia inducible factor subunit, HIF3 $\alpha$. Gene Expr. 7: 205-213.

Gu, Y.Z., Hogenesch, J.B., and Bradfield, C.A. 2000. The PAS superfamily: Sensors of environmental and developmental signals. Annu. Rev. Pharmacol. Toxicol. 40: 519-561.

Haase, V.H., Glickman, J.N., Socolovsky, M., and Jaenisch, R. 2001. Vascular tumors in livers with targeted inactivation of the von Hippel-Lindau tumor suppressor. Proc. Natl. Acad. Sci. 98: 1583-1588.

Heacock, C.S. and Sutherland, R.M. 1990. Enhanced synthesis of stress proteins caused by hypoxia and relation to altered cell growth and metabolism. Br. J. Cancer 62: 217-225.

Hewitson, K.S., McNeill, L.A., Riordan, M.V., Tian, Y.M., Bullock, A.N., Welford, R.W., Elkins, J.M., Oldham, N.J., Bhattacharya, S., Gleadle, J.M., et al. 2002. Hypoxia-inducible factor (HIF) asparagine hydroxylase is identical to factor inhibiting HIF (FIH) and is related to the cupin structural family. J. Biol. Chem. 277: 26351-26355.

Hogenesch, J.B., Chan, W.K., Jackiw, V.H., Brown, R.C., Gu, Y.Z., Pray-Grant, M., Perdew, G.H., and Bradfield, C.A. 1997. Characterization of a subset of the basic-helix-loophelix-PAS superfamily that interacts with components of the dioxin signaling pathway. J. Biol. Chem. 272: 8581-8593.

Hu, C.J., Wang, L.Y., Chodosh, L.A., Keith, B., and Simon, M.C. 2003. Differential roles of hypoxia-inducible factor $1 \alpha$ (HIF$1 \alpha)$ and HIF- $2 \alpha$ in hypoxic gene regulation. Mol. Cell. Biol. 23: $9361-9374$.

Huang, Y., Hickey, R.P., Yeh, J.L., Liu, D., Dadak, A., Young, L.H., Johnson, R.S., and Giordano, F.J. 2004. Cardiac myocyte-specific HIF- $1 \alpha$ deletion alters vascularization, energy availability, calcium flux, and contractility in the normoxic heart. FASEB J. 18: 1138-1140.

Katschinski, D.M., Marti, H.H., Wagner, K.F., Shibata, J., Eckhardt, K., Martin, F., Depping, R., Paasch, U., Gassmann, M., Ledermann, B., et al. 2003. Targeted disruption of the mouse PAS domain serine/threonine kinase PASKIN. Mol. Cell. Biol. 23: 6780-6789.

Kondo, K., Kim, W.Y., Lechpammer, M., and Kaelin Jr., W.G., 2003. Inhibition of HIF2a is sufficient to suppress pVHLdefective tumor growth. PLOS Biol. 1: E83.

Koumenis, C., Naczki, C., Koritzinsky, M., Rastani, S., Diehl, A., Sonenberg, N., Koromilas, A., and Wouters, B.G. 2002. Regulation of protein synthesis by hypoxia via activation of the endoplasmic reticulum kinase PERK and phosphoryla- tion of the translation initiation factor eIF2 $\alpha$. Mol. Cell. Biol. 22: $7405-7416$.

Lando, D., Peet, D.J., Gorman, J.J., Whelan, D.A., Whitelaw, M.L., and Bruick, R.K. 2002a. FIH-1 is an asparaginyl hydroxylase enzyme that regulates the transcriptional activity of hypoxia-inducible factor. Genes \& Dev. 16: 1466-1471.

Lando, D., Peet, D.J., Whelan, D.A., Gorman, J.J., and Whitelaw, M.L. 2002b. Asparagine hydroxylation of the HIF transactivation domain a hypoxic switch. Science 295: 858-861.

Lopez-Barneo, J., del Toro, R., Levitsky, K.L., Chiara, M.D., and Ortega-Saenz, P. 2004. Regulation of oxygen sensing by ion channels. J. Appl. Physiol. 96: 1187-1195; discussion 11701182.

Mack, F.A., Rathmell, W.K., Arsham, A.M., Gnarra, J., Keith, B., and Simon, M.C. 2003. Loss of pVHL is sufficient to cause HIF dysregulation in primary cells but does not promote tumor growth. Cancer Cell 3: 75-88.

Mahon, P.C., Hirota, K., and Semenza, G.L. 2001. FIH-1: A novel protein that interacts with HIF- $1 \alpha$ and VHL to mediate repression of HIF-1 transcriptional activity. Genes \& Dev. 15: $2675-2686$.

Makino, Y., Cao, R., Svensson, K., Bertilsson, G., Asman, M., Tanaka, H., Cao, Y., Berkenstam, A., and Poellinger, L. 2001. Inhibitory PAS domain protein is a negative regulator of hypoxia-inducible gene expression. Nature 414: 550-554.

Maltepe, E., Schmidt, J.V., Baunoch, D., Bradfield, C.A., and Simon, M.C. 1997. Abnormal angiogenesis and responses to glucose and oxygen deprivation in mice lacking the protein ARNT. Nature 386: 403-407.

Maxwell, P.H., Wiesener, M.S., Chang, G.W., Clifford, S.C., Vaux, E.C., Cockman, M.E., Wykoff, C.C., Pugh, C.W., Maher, E.R., and Ratcliffe, P.J. 1999. The tumour suppressor protein VHL targets hypoxia-inducible factors for oxygendependent proteolysis. Nature 399: 271-275.

McMurtry, I.F., Bauer, N.R., Fagan, K.A., Nagaoka, T., Gebb, S.A., and Oka, M. 2003. Hypoxia and Rho/Rho-kinase signaling. Lung development versus hypoxic pulmonary hypertension. Adv. Exp. Med. Biol. 543: 127-137.

McNeill, L.A., Hewitson, K.S., Claridge, T.D., Seibel, J.F., Horsfall, L.E., and Schofield, C.J. 2002. Hypoxia-inducible factor asparaginyl hydroxylase (FIH-1) catalyses hydroxylation at the $\beta$-carbon of Asparagine-803. Biochem. J. 367: 571-575.

Mekhail, K., Gunaratnam, L., Bonicalzi, M.E., and Lee, S. 2004. HIF activation by $\mathrm{pH}$-dependent nucleolar sequestration of VHL. Nat. Cell. Biol. 6: 642-647.

Miao, W., Luo, Z., Kitsis, R.N., and Walsh, K. 2000. Intracoronary, adenovirus-mediated Akt gene transfer in heart limits infarct size following ischemia-reperfusion injury in vivo. $J$. Mol. Cell. Cardiol. 32: 2397-2402.

Minamino, T. and Kourembanas, S. 2001. Mechanisms of telomerase induction during vascular smooth muscle cell proliferation. Circ. Res. 89: 237-243.

Minamino, T., Christou, H., Hsieh, C.M., Liu, Y., Dhawan, V., Abraham, N.G., Perrella, M.A., Mitsialis, S.A., and Kourembanas, S. 2001. Targeted expression of heme oxygenase-1 prevents the pulmonary inflammatory and vascular responses to hypoxia. Proc. Nat1. Acad. Sci. 98: 8798-8803.

Moeller, B.J., Cao, Y., Li, C.Y., and Dewhirst, M.W. 2004. Radiation activates HIF-1 to regulate vascular radiosensitivity in tumors: Role of reoxygenation, free radicals, and stress granules. Cancer Cell 5: 429-441.

Ohh, M., Park, C.W., Ivan, M., Hoffman, M.A., Kim, T.Y., Huang, L.E., Pavletich, N., Chau, V., and Kaelin, W.G. 2000. Ubiquitination of hypoxia-inducible factor requires direct binding to the $\beta$-domain of the von Hippel-Lindau protein. Nat. Cell. Biol. 2: 423-427. 
Park, S.K., Dadak, A.M., Haase, V.H., Fontana, L., Giaccia, A.J., and Johnson, R.S. 2003. Hypoxia-induced gene expression occurs solely through the action of hypoxia-inducible factor $1 \alpha$ (HIF-1 $\alpha$ ): Role of cytoplasmic trapping of HIF- $2 \alpha$. Mol. Cell. Biol. 23: 4959-4971.

Peng, Y.J. and Prabhakar, N.R. 2004. Effect of two paradigms of chronic intermittent hypoxia on carotid body sensory activity. J. Appl. Physiol. 96: 1236-1242; discussion 1196.

Peng, J., Zhang, L., Drysdale, L., and Fong, G.H. 2000. The transcription factor EPAS-1/hypoxia-inducible factor $2 \alpha$ plays an important role in vascular remodeling. Proc. Natl. Acad. Sci. 97: 8386-8391.

Potter, C. and Harris, A.L. 2004. Hypoxia inducible carbonic anhydrase IX, marker of tumour hypoxia, survival pathway and therapy target. Cell Cycle 3: 164-167.

Raj, U. and Shimoda, L. 2002. Oxygen-dependent signaling in pulmonary vascular smooth muscle. Am. J. Physiol. Lung Cell. Mol. Physiol. 283: L671-L677.

Rapisarda, A., Uranchimeg, B., Scudiero, D.A., Selby, M., Sausville, E.A., Shoemaker, R.H., and Melillo, G. 2002. Identification of small molecule inhibitors of hypoxia-inducible factor 1 transcriptional activation pathway. Cancer Res. 62: 4316-4324.

Rapisarda, A., Uranchimeg, B., Sordet, O., Pommier, Y., Shoemaker, R.H., and Melillo, G. 2004. Topoisomerase I-mediated inhibition of hypoxia-inducible factor 1: Mechanism and therapeutic implications. Cancer Res. 64: 1475-1482.

Rini, B.I., Halabi, S., Taylor, J., Small, E.J., and Schilsky, R.L. 2004. Cancer and Leukemia Group B 90206: A randomized phase III trial of interferon- $\alpha$ or interferon- $\alpha$ plus anti-vascular endothelial growth factor antibody (bevacizumab) in metastatic renal cell carcinoma. Clin. Cancer Res. 10: 25842586.

Schipani, E., Ryan, H.E., Didrickson, S., Kobayashi, T., Knight, M., and Johnson, R.S. 2001. Hypoxia in cartilage: HIF- $1 \alpha$ is essential for chondrocyte growth arrest and survival. Genes \& Dev. 15: 2865-2876.

Schofield, C.J. and Ratcliffe, P.J. 2004. Oxygen sensing by HIF hydroxylases. Nat. Rev. Mol. Cell. Biol. 5: 343-354.

Schroedl, C., McClintock, D.S., Budinger, G.R., and Chandel, N.S. 2002. Hypoxic but not anoxic stabilization of HIF-1a requires mitochondrial reactive oxygen species. Am. I. Physiol. Lung Cell. Mol. Physiol. 283: L922-L931.

Schumacker, P.T. 2003. Current paradigms in cellular oxygen sensing. Adv. Exp. Med. Biol. 543: 57-71.

Scortegagna, M., Ding, K., Oktay, Y., Gaur, A., Thurmond, F., Yan, L.J., Marck, B.T., Matsumoto, A.M., Shelton, J.M., Richardson, J.A., et al. 2003. Multiple organ pathology, metabolic abnormalities and impaired homeostasis of reactive oxygen species in Epas $1^{-/-}$mice. Nat. Genet. 35: 331340.

Semenza, G.L. 2003. Targeting HIF-1 for cancer therapy. Nat. Rev. Cancer 3: 721-732.

—. 2004. $\mathrm{O}_{2}$-regulated gene expression: Transcriptional control of cardiorespiratory physiology by HIF-1. I. Appl. Physiol. 96: 1173-1177; discussion 1170-1172.

Siemann, D.W., Chaplin, D.J., and Horsman, M.R. 2004. Vascular-targeting therapies for treatment of malignant disease. Cancer 100: 2491-2499.

Spencer, T.E., Jenster, G., Burcin, M.M., Allis, C.D., Zhou, J., Mizzen, C.A., McKenna, N.J., Onate, S.A., Tsai, S.Y., Tsai, M.J., et al. 1997. Steroid receptor coactivator-1 is a histone acetyltransferase. Nature 389: 194-198.

Sutphin, P.D., Chan, D.A., and Giaccia, A.J. 2004. Dead cells don't form tumors: HIF-dependent cytotoxins. Cell Cycle 3: $160-163$.
Tian, H., McKnight, S.L., and Russell, D.W. 1997. Endothelial PAS domain protein 1 (EPAS1), a transcription factor selectively expressed in endothelial cells. Genes \& Dev. 11: 7282.

Tian, H., Hammer, R.E., Matsumoto, A.M., Russell, D.W., and McKnight, S.L. 1998. The hypoxia-responsive transcription factor EPAS1 is essential for catecholamine homeostasis and protection against heart failure during embryonic development. Genes \& Dev. 12: 3320-3324.

Vaupel, P. 2004. Tumor microenvironmental physiology and its implications for radiation oncology. Semin. Radiat. Oncol. 14: 198-206.

Vogel, J., Kiessling, I., Heinicke, K., Stallmach, T., Ossent, P., Vogel, O., Aulmann, M., Frietsch, T., Schmid-Schonbein, H., Kuschinsky, W., et al. 2003. Transgenic mice overexpressing erythropoietin adapt to excessive erythrocytosis by regulating blood viscosity. Blood 102: 2278-2284.

Wang, G.L. and Semenza, G.L. 1995. Purification and characterization of hypoxia-inducible factor 1. J. Biol. Chem. 270: 1230-1237.

Waypa, G.B. and Schumacker, P.T. 2002. O(2) sensing in hypoxic pulmonary vasoconstriction: The mitochondrial door re-opens. Respir. Physiol. Neurobiol. 132: 81-91.

Yun, Z., Maecker, H.L., Johnson, R.S., and Giaccia, A.J. 2002. Inhibition of PPARg2 gene expression by the HIF-1-regulated gene DEC1/Stra13. A mechanism for regulation of adipogenesis by hypoxia. Dev. Cell 2: 331-341. 


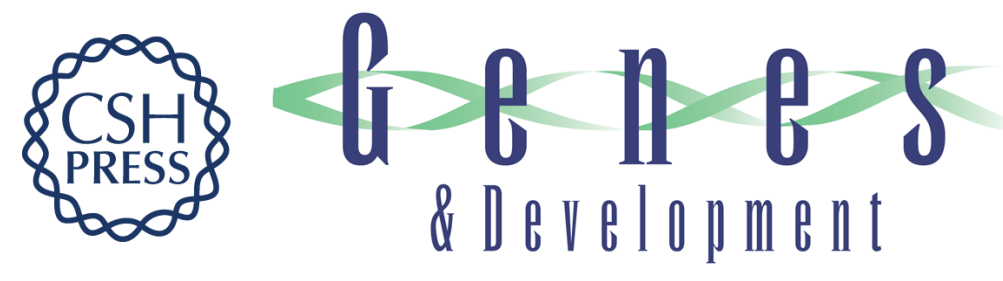

\section{The biology of hypoxia: the role of oxygen sensing in development, normal function, and disease}

Amato J. Giaccia, M. Celeste Simon and Randall Johnson

Genes Dev. 2004, 18:

Access the most recent version at doi:10.1101/gad.1243304

References This article cites 68 articles, 31 of which can be accessed free at: http://genesdev.cshlp.org/content/18/18/2183.full.html\#ref-list-1

License

Email Alerting

Receive free email alerts when new articles cite this article - sign up in the box at the top Service right corner of the article or click here.

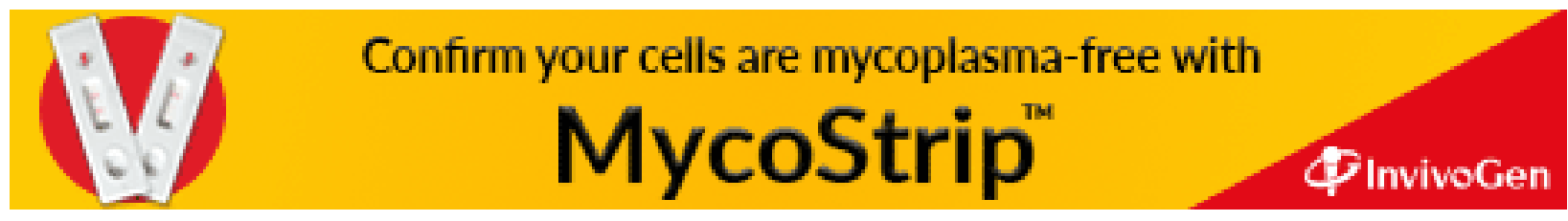

